\title{
External Examination
}

National Cancer Institute

\section{Source}

National Cancer Institute. External Examination. NCI Thesaurus. Code C124596.

An assessment of the outside of the body. 